\begin{tabular}{|l|c|c|c|c|}
\hline $\begin{array}{l}\text { Cuadernos de Investigación Geográfica } \\
\text { Geographical Research Letters }\end{array}$ & 2019 & N $^{\circ} 45(1)$ & pp.61-86 & eISSN 1697-9540 \\
\hline
\end{tabular}

\title{
LA INFLUENCIA HUMANA, CLAVE PARA ENTENDER LA BIOGEOGRAFÍA DE LAS ESPECIES INVASORAS EN EL ANTROPOCENO
}

\author{
B. GALLARDO*, L. VILA
}

\begin{abstract}
Instituto Pirenaico de Ecología, Consejo Superior de Investigaciones Científicas (IPE-CSIC), Campus de Aula Dei, Apartado 13.034, 50080 Zaragoza, Spain.
\end{abstract}

RESUMEN. Las invasiones biológicas han revolucionado el concepto de biogeografía en el Antropoceno, ya que su distribución geográfica ya no depende de su capacidad intrínseca de movimiento y las barreras biogeográficas clásicas, sino que depende directamente de actividades humanas que favorecen, intencionada o accidentalmente, su expansión. Por tanto, las técnicas de modelización geográfica han de tener en cuenta las particularidades de las especies invasoras para poder anticipar su distribución potencial en el futuro. En este estudio, utilizamos un listado de 57 de las peores especies invasoras en Europa (18 plantas, 15 vertebrados, 12 invertebrados y 12 organismos acuáticos) para comparar la influencia climática y humana en la distribución a gran escala de especies invasoras. Identificamos un total de nueve grandes vectores de introducción, entre los que destacan el transporte, el cultivo ornamental y el comercio. Localizamos siete variables relacionadas con estos vectores de introducción que utilizamos junto con variables climáticas como predictores en Modelos Ecológicos de Nicho (MEN): accesibilidad, densidad de población, $P I B$, grado de antropización, cobertura de uso agrícola y urbano, distancia a carreteras y puertos comerciales. La temperatura mínima anual es el predictor más importante en los modelos, seguida de la distancia a puertos, el PIB y la accesibilidad. Sorprendentemente, los modelos que incluyen variables humanas no son estadísticamente mejores que los basados en variables climáticas únicamente, si bien predicen un aumento medio del $8 \%$ en la superficie europea susceptible a la invasión. Tal incremento se produce fundamentalmente en zonas muy antropizadas donde la posibilidad de invasión es obviamente mayor. En el caso de la Península Ibérica, esto se traduce en un aumento del riesgo de invasión en la zona de levante, valle del Ebro y alrededores de grandes ciudades. Concluimos por tanto que el clima es importante, pero no suficiente para anticipar efectivamente la expansión de especies invasoras, y por tanto, cualquier información disponible que esté directamente relacionada con los vectores de introducción ha de ser incorporada de forma rutinaria en los MEN para optimizar los recursos dedicados a la prevención, respuesta rápida y control a largo plazo de especies invasoras. 


\section{Human influence, key to understand the biogeography of invasive species in the Anthropocene}

ABSTRACT. Biological invasions have challenged the traditional concept of biogeography in the Anthropocene. This is because the geographical distribution of invasive species is no longer dependent on its movement ability and the presence of biogeographical barriers, rather on the human activities that promote the expansion of invasive species, intentionally or accidentally. Consequently, modelling techniques must take into account the idiosyncrasies of invasive species in order to effectively anticipate their potential distribution. In this study we use as reference a list of 57 of the worst invasive species in Europe (18 plants, 15 vertebrates, 12 invertebrates and 12 aquatic organisms) to compare the influence of climate and human activities on the large-scale distribution of invaders. We identified nine major vectors of distribution, highlighting transport, ornamental use and trade. We also located seven human variables that we could use as surrogates of those vectors in Ecological Niche Models (ENM): accessibility, population density, GDP, human influence index, urban and agriculture cover, distance to roads and commercial ports. Minimum annual temperature was the most important predictor in models integrating climatic and human variables, followed by distance to ports, GDP and accessibility. Surprisingly, integrated models were not statistically better than models based on climate variables only, yet they anticipated an average increase of $8 \%$ in the European surface susceptible to invasion. Such increment is especially notorious in areas under a high human influence where we can presume a higher propagule pressure. In the Iberian Peninsula, the integrated model suggests a higher risk of invasion than the climate model in the eastern coast, Ebro valley and surroundings of major cities. We conclude that climate is important, but not enough to effectively anticipate the spread of invasive species and thus information directly related with the vectors of species introduction must be included routinely in ENM to optimize resources invested in the prevention, rapid response and long-term control of invasive species.

Palabras clave: Vector de introducción, modelos de distribución de especies, modelos ecológicos de nicho, presión de propágulos.

Key words: Introduction vectors, species distribution models, ecological niche models, propagule pressure.

Recibido: 24 de mayo de 2018

Aceptado: 27 de septiembre de 2018

*Correspondencia: Belinda Gallardo, Instituto Pirenaico de Ecología, Consejo Superior de Investigaciones Científicas (IPE-CSIC), Campus de Aula Dei, Apartado 13.034, 50080 Zaragoza, España. E-mail: Belinda@ipe.csic.es 


\section{Introducción}

Durante las últimas décadas, los impactos ecológicos y humanos causados por el asentamiento de especies invasoras han generado una creciente preocupación e interés por conocer su dispersión y ahondar en sus factores determinantes. En condiciones naturales, factores bióticos (i.e. interacción entre especies), abióticos (i.e. clima) y las barreras biogeográficas (i.e. cadenas montañosas, océanos), determinan el potencial de establecimiento y expansión de una especie (diagrama BAM, Soberón y Nakamura, 2009) (Fig. 1A). Sin embargo, en el contexto actual, la influencia que ejercen el hombre y sus actividades han permitido la expansión de especies más allá de sus rangos de dispersión naturales (Seebens et al., 2018). Las invasiones biológicas rompen de este modo las barreras biogeográficas clásicas, y su distribución espacial a gran escala es resultado del transporte de especies a través de actividades desarrolladas por el ser humano, redefiniendo el concepto de biogeografía en el Antropoceno (Capinha et al., 2015). Es más, el ritmo al que se descubren nuevas especies invasoras no deja de crecer (Seebens et al., 2017), lo que aumenta progresivamente la similitud entre las comunidades de flora y fauna de regiones muy alejadas entre sí, con importantes consecuencias ecológicas y evolutivas (Olden et al., 2004) y seguramente también en el uso de los recursos naturales por parte del hombre (Olden et al., 2005).

A) Diagrama BAM nativas

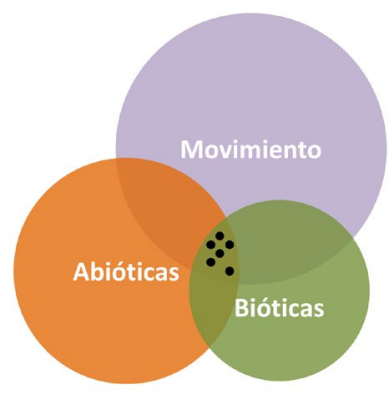

B) Diagrama BAM invasoras

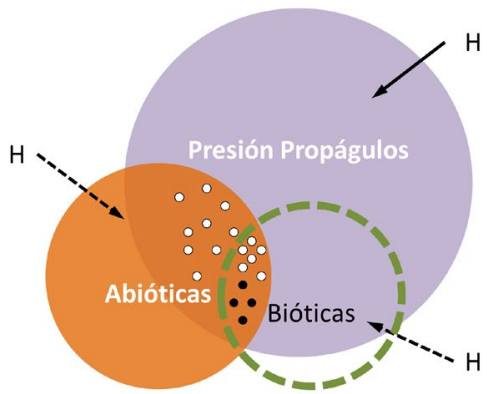

Figura 1. Factores que determinan el nicho ecológico de una especie. A) Diagrama BAM que ilustra como la combinación de factores Bióticos (competencia, depredación, parasitismo), Abióticos (clima, condiciones de hábitat) y de Movimiento (capacidad de dispersión, localización de barreras biogeográficas) determinan la distribución histórica de las especies nativas, representada por puntos negros (modificado de Soberón y Nakamura, 2009). B) En el caso de las especies invasoras, la actividad humana $(H)$ modifica los condicionantes del nicho ecológico. Las especies invasoras a menudo carecen de limitaciones biológicas y su movimiento deriva de vectores humanos (e.g. comercio, transporte), por lo que su distribución invasora, representada por puntos blancos, depende casi exclusivamente de la combinación entre la presión de propágulos y las condiciones abióticas.

Las especies invasoras se definen según el Convenio sobre la Diversidad Biológica (CBD, https://www.cbd.int/invasive/) como aquellos taxones cuya introducción fuera de su área de distribución natural por medio del hombre constituye una amenaza para la biodiversidad. Las especies invasoras se diferencian por tanto de las nativas en que su distribución ya 
no está limitada por su capacidad intrínseca de movimiento y las barreras biogeográficas que se puedan encontrar, sino que depende de la "presión de propágulos", es decir, del número de individuos y frecuencia con que se introduce una nueva especie en una región (Lockwood et al., 2005). Es más, las especies invasoras a menudo poseen rasgos biológicos muy competitivos (Van Kleunen et al., 2010; Estrada et al., 2016), carecen de las habituales limitaciones bióticas (e.g. depredadores, competidores o parásitos) de su rango nativo (Callaway y Ridenour, 2004), y tienden a establecer nuevas interacciones en el área invadida (e.g. nuevas presas), de modo que la influencia del factor biótico se diluye (Tylianakis et al., 2008). Esto nos obliga a reformular la idea tradicional de factores limitantes del nicho ecológico (Fig. 1A), que en el caso de las especies invasoras acaba únicamente determinado por las preferencias climáticas básicas de la especie y su presión de propágulos (Fig. 1B) (Capinha et al., 2015).

Los Modelos Ecológicos de Nicho (MEN) ofrecen una herramienta óptima para el estudio de escenarios en la distribución de especies invasoras en el Antropoceno. Los MEN relacionan la presencia de una especie con las condiciones ambientales donde se encuentra presente, generando un modelo de probabilidad de establecimiento (Guisan y Thuiller, 2005). Estos modelos son utilizados posteriormente para localizar las áreas geográficas con condiciones ambientales similares a las zonas ocupadas hasta el momento, y en las que por tanto podemos esperar un mayor riesgo de establecimiento y supervivencia en caso de que la especie sea introducida. Tradicionalmente, los MEN solamente tomaban en consideración factores climáticos, los cuales determinan aceptablemente la distribución de especies a escala global y regional (Hijmans y Graham, 2006). Sin embargo, tal y como se han venido utilizando, estos modelos subestiman el papel de la influencia humana, y más en particular de la presión de propágulos, en la distribución actual y futura de las especies invasoras (Gallardo et al., 2015). Podemos argumentar que una región con alta idoneidad climática para una especie invasora nunca será colonizada, o las probabilidades serán muy bajas, sino existe un vector que facilite la llegada de la especie. Por el contrario, si la presión de propágulos es muy elevada, cabe esperar que la especie acabe estableciéndose aun cuando las condiciones ambientales no sean las óptimas.

Efectivamente, aquellos estudios que han considerado factores relacionados con la presión de propágulos -mediante variables como el Índice de Influencia Humana (Sanderson et al., 2002), la densidad de población, el uso del suelo y la distancia a puertos-coinciden en destacar la capacidad predictiva y credibilidad de las predicciones (Gallardo y Aldridge, 2013; Gallardo et al., 2015; Zhu et al., 2016; Gallardo et al.,2017). Este tipo de modelos que integran información climática y humana, permiten ahondar en los factores determinantes de las invasiones biológicas y su posible evolución en el futuro (Gallardo et al., 2018). El aumento de investigaciones que incluyen la dimensión humana en los últimos años se debe en gran medida al desarrollo de las tecnologías de información geográfica y "big data”, que hace accesible una gran cantidad de información a escala global y permiten calibrar modelos de una complejidad sin precedentes (Hampton et al., 2013). No obstante, la mayoría de los estudios hasta el momento se centra en una o pocas especies, y utilizando un número mas bien limitado de indicadores, lo que impide realizar generalizaciones y comparaciones a través de grupos taxonómicos.

El objetivo principal de este trabajo es evaluar el efecto de la presión de propágulos sobre la distribución global de especies invasoras. Para ello, tomamos como referencia el listado de las "peores especies invasoras" en Europa (http://www.europe-aliens.org/). El listado 
incluye especies de multiples grupos taxonómicos, con diversos rasgos biológicos, vectores de introducción, impactos y fases de invasión, que representan el amplio abanico de especies invasoras que nos podemos encontrar en Europa. El abordar este estudio desde un punto de vista multiespecie, nos permite comparar patrones entre hábitats y grupos taxonómicos.

Este estudio gira en torno a tres hipótesis fundamentales:

1. El hombre juega un papel determinante en la distribución actual de especies invasoras, por tanto los MEN que incluyen actividades humanas relacionadas con la presión de propágulos ofrecerán una mayor calidad y capacidad predictiva que modelos basados únicamente en variables climáticas.

2. Si esto es así, es de esperar que los modelos climáticos subestimen el potencial de invasión en zonas con elevada influencia humana (i.e. menor idoneidad de hábitat del modelo climático).

3. Por el contrario, los modelos climáticos son susceptibles de sobreestimar el potencial invasor en regiones relativamente prístinas y alejadas de la influencia humana, donde las probabilidades de que la especie sea introducida son más bien bajas, independientemente de su idoneidad climática (i.e. mayor idoneidad de hábitat del modelo climático).

\section{Métodos}

\subsection{Datos de presencia de especies invasoras}

En este estudio nos centramos en 57 de las peores especies invasoras identificadas por expertos de toda Europa en el marco del proyecto DAISIE (http://www .europe-aliens. org/). Este listado incluye: 12 especies de agua dulce, 15 vertebrados, 12 invertebrados terrestres y 18 plantas (Tabla 1). Treinta y tres de ellas están incluidas en el Catálogo Español de Especies Exóticas Invasoras (https://goo.gl/Bttuwz) y catorce en el Listado de Especies Exóticas Invasoras preocupantes para la Unión Europea (https://goo.gl/fwhvjr).

Para realizar los modelos, partimos de los datos globales de presencia de las 57 especies invasoras reunidos por Gallardo et al. (2017), que a su vez fueron recopilados a partir de la consulta de diversas bases de datos globales, tales como:

- Compendio de Especies Invasoras de CABI (CABI-ISC, www .cabi.org)

- Infraestructura Mundial de Información sobre Biodiversidad (GBIF, www .gbif.es)

- Unión Internacional para la Conservación de la Naturaleza (IUCN, www.iucn.org)

- Biocolecciones integradas en formato digital de la National Science Foundation (www.idigbio.org)

- Servicio geológico de los Estados Unidos (www.usgs.gov)

- $\quad$ Red de información global de especies invasoras (www.gisin.org)

- Datos recopilados por la Encyclopedia of life (www.eol.org) 
Tras haber limpiado los datos para eliminar coordenadas erróneas y duplicados (más de una presencia por cuadrícula de $1 \times 1 \mathrm{~km}$ ), la base de datos de presencias incluyó un total de 217.000 datos, con una media de 3700 presencias globales por especie (Tabla 1).

Tabla 1. Especies invasoras en Europa consideradas en este estudio incluyendo grupo, nombre científico, región nativa de origen, número total de datos georreferenciados utilizados en posteriores análisis estadísticos $(N)$ y principales vectores de introducción en Europa. Las especies marcadas con * están incluidas en el Catálogo Español de Especies Exóticas Invasoras.

\begin{tabular}{|c|c|c|c|c|c|c|c|c|c|c|c|c|}
\hline Grupo & Nombre científico & Región nativa & $\mathbf{N}$ & 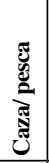 & 苞 &  & 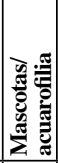 & 焉 &  & 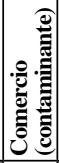 & 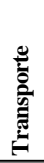 & 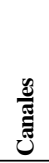 \\
\hline \multirow{13}{*}{ 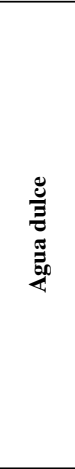 } & Anguillicola crassus & Asia & 116 & & & & & & & & & \\
\hline & Cercopagis pengoi & Europa & 146 & & & & & & & & & \\
\hline & Corbicula fluminea* & Asia & 2333 & & & & & & & & & \\
\hline & Cordylophora caspia* & Europa & 143 & & & & & & & & & \\
\hline & Crassula helmsii & Australia & 1483 & & & & & & & & & \\
\hline & Dikerogammarus villosus & Europa & 498 & & & & & & & & & \\
\hline & Dreissena polymorpha* & Europa & 3029 & & & & & & & & & \\
\hline & Elodea canadensis* & América del Norte & 10463 & & & & & & & & & \\
\hline & Eriocheir sinensis* & Asia & 443 & & & & & & & & & \\
\hline & Neogobius melanostomus & Europa & 482 & & & & & & & & & \\
\hline & Procambarus clarkii ${ }^{*}$ & América del Norte & 886 & & & & & & & & & \\
\hline & Pseudorasbora parva* & Asia & 878 & & & & & & & & & \\
\hline & Salvelinus fontinalis* & América del Norte & 3068 & & & & & & & & & \\
\hline \multirow{11}{*}{ 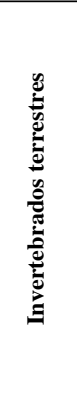 } & Aedes albopictus* & Asia & 1410 & & & & & & & & & \\
\hline & Arion vulgaris & Europa & 835 & & & & & & & & & \\
\hline & Bemisia tabaci & Desconocido & 425 & & & & & & & & & \\
\hline & Cameraria ohridella & Desconocido & 1245 & & & & & & & & & \\
\hline & Ceratitis capitata & África & 705 & & & & & & & & & \\
\hline & Diabrotica virgifera & América Central & 583 & & & & & & & & & \\
\hline & Harmonia axyridis ${ }^{*}$ & Asia & 2285 & & & & & & & & & \\
\hline & Leptinotarsa decemlineata & América Central & 508 & & & & & & & & & \\
\hline & Linepithema humile* $^{*}$ & América del Sur & 1215 & & & & & & & & & \\
\hline & Liriomyza huidobrensis & América del Sur & 403 & & & & & & & & & \\
\hline & Spodoptera littoralis & África & 113 & & & & & & & & & \\
\hline \multirow{12}{*}{ 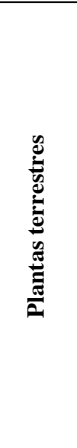 } & Acacia dealbata* & Australia & 1277 & & & & & & & & & \\
\hline & Ailanthus altissima* & Asia & 2566 & & & & & & & & & \\
\hline & Ambrosia artemisiifolia* & América del Norte & 5212 & & & & & & & & & \\
\hline & Campylopus introflexus & América del Sur & 6143 & & & & & & & & & \\
\hline & Carpobrotus edulis* & África & 773 & & & & & & & & & \\
\hline & Cortaderia selloana* & América del Sur & 999 & & & & & & & & & \\
\hline & Echinocystis lobata & América del Norte & 1385 & & & & & & & & & \\
\hline & Fallopia japonica* ${ }^{*}$ & Asia & 10723 & & & & & & & & & \\
\hline & Hedychium gardnerianum* & Asia & 121 & & & & & & & & & \\
\hline & $\begin{array}{l}\text { Heracleum mantegazzia- } \\
\text { num* }\end{array}$ & Asia & 5633 & & & & & & & & & \\
\hline & Impatiens glandulifera & Asia & 8059 & & & & & & & & & \\
\hline & Opuntia ficus-indica* & América Central & 369 & & & & & & & & & \\
\hline
\end{tabular}




\begin{tabular}{|c|c|c|c|c|c|c|c|c|c|c|c|c|}
\hline Grupo & Nombre científico & Región nativa & $\mathbf{N}$ & 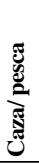 & 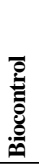 & 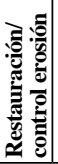 & 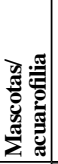 & 焉 & 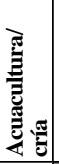 & 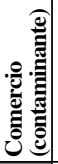 & & 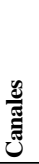 \\
\hline & Oxalis pes-caprae* & África & 1397 & & & & & & & & & \\
\hline & Paspalum paspalodes & América Central & 2150 & & & & & & & & & \\
\hline & Prunus serotina & América del Norte & 6946 & & & & & & & & & \\
\hline & Rhododendron ponticum & Europa & 3182 & & & & & & & & & \\
\hline & Robinia pseudoacacia & América del Norte & 9365 & & & & & & & & & \\
\hline & Rosa rugosa & Asia & 5261 & & & & & & & & & \\
\hline \multirow{15}{*}{  } & Branta canadensis* & América del Norte & 40320 & & & & & & & & & \\
\hline & Cervus nippon & Asia & 1214 & & & & & & & & & \\
\hline & Lithobates catesbeianus* & América del Norte & 4510 & & & & & & & & & \\
\hline & Mustela vison* & América del Norte & 5301 & & & & & & & & & \\
\hline & Myocastor coypus* & América del Sur & 2745 & & & & & & & & & \\
\hline & Nyctereutes procyonoides $*$ & Asia & 1772 & & & & & & & & & \\
\hline & Ondatra zibethicus* & América del Norte & 3152 & & & & & & & & & \\
\hline & Oxyura jamaicensis ${ }^{*}$ & América del Norte & 17144 & & & & & & & & & \\
\hline & Procyon lotor $*$ & América del Norte & 15468 & & & & & & & & & \\
\hline & Psittacula krameri ${ }^{*}$ & África & 1279 & & & & & & & & & \\
\hline & Rattus norvegicus* & Europa & 9825 & & & & & & & & & \\
\hline & Sciurus carolinensis $*$ & América del Norte & 5166 & & & & & & & & & \\
\hline & Tamias sibiricus & Asia & 131 & & & & & & & & & \\
\hline & Threskiornis aethiopicus* & África & 2347 & & & & & & & & & \\
\hline & Trachemys scripta* & América del Norte & 1503 & & & & & & & & & \\
\hline
\end{tabular}

\subsection{Vías de introducción de especies invasoras}

Realizamos una extensiva búsqueda bibliográfica para determinar los principales vectores de introducción de cada una de las 57 especies invasoras, utilizando la clasificación desarrollada por la European Alien Species Information Network (EASIN) (Katsanevakis et al., 2012) (Tabla 1).

Una vez determinados, buscamos capas geográficas (ráster y vectoriales) que pudieran ser utilizadas como proxies directos o indirectos asociados a cada vector (Tabla S1). A pesar de que existen multitud de mapas temáticos para distintas regiones del mundo (e.g. mapas disponibles en la IDE de España), todos los autores coinciden en la necesidad de modelizar a las especies invasoras teniendo en cuenta su rango de distribución global (Broennimann y Guisan, 2008), por lo que es fundamental contar con capas geográficas a esta escala. Una de nuestras mayores limitaciones fue por tanto encontrar capas con cobertura (global) y resolución (máximo 5 arcominutos, unos 10x10 km en el ecuador) suficientes como para ser utilizadas posteriormente en los MEN.

En la Tabla S1 se recoge un listado de las 23 capas de partida que consideramos, con información acerca de su cobertura, resolución, orígen y relación con los vectores de introducción de las especies invasoras listados en la Tabla 1. Para evitar problemas de collinearidad en posteriores análisis estadísticos, generamos una matriz de correlación con las capas de influencia humana. En el caso de capas con una correlación superior a 0,7 , nos quedamos con 
la que tuviera una relación más directa con la introducción de especies invasoras y causara menos problemas de correlación con otras capas. Este fue el caso de pesca y acuacultura $(\mathrm{r}=$ $0,76)$, pastos y praderas $(r=0,95)$. Algunas capas fueron descartadas por problemas de cobertura o resolución (e.g. fragmentación de ríos o distancia a embalses solo disponible a 30'). La Tabla 2 recoge las 7 variables humanas finalmente incluidas en los modelos. La resolución de todas las capas se transformó a 5 arco-minutos antes de realizar los análisis.

Tabla 2. Variables incluidas en el modelo climático (Bio5-Bio15) y modelo integrado (todas). Todas las capas tienen una cobertura global. Res.: resolución original de la capa. A nivel del ecuador, 30" equivalen aproximadamente a $1 \times 1 \mathrm{~km}$. Todas las capas fueron transformadas a 5 , de resolución (10x10 km aprox.).

\begin{tabular}{|c|c|c|c|c|}
\hline Variable & Unidades & $\operatorname{Res}$ & Descripción & Fuente \\
\hline Bio 5 & ${ }^{\circ} \mathrm{C}$ & $30^{\prime \prime}$ & $\mathrm{T}^{\mathrm{a}}$ máxima del mes más cálido & Global Climate Data (http://www.worldclim.org/) \\
\hline Bio 6 & ${ }^{\circ} \mathrm{C}$ & $30 "$ & $\mathrm{~T}^{\mathrm{a}}$ mínima del mes más frío & \\
\hline Bio13 & $\mathrm{mm}$ & $30 "$ & $\begin{array}{l}\text { Precipitación del mes más } \\
\text { húmedo }\end{array}$ & \\
\hline Bio 14 & $\mathrm{~mm}$ & $30 "$ & Precipitación del mes más seco & \\
\hline Bio 15 & $\mathrm{~mm}$ & $30 "$ & $\begin{array}{l}\text { Estacionalidad de la } \\
\text { precipitación }\end{array}$ & \\
\hline $\begin{array}{l}\text { Accesibil- } \\
\text { idad }\end{array}$ & Días/h & $30^{\prime \prime}$ & $\begin{array}{l}\text { Tiempo de viaje a la ciudad de } \\
>50.000 \text { habitantes más cercana }\end{array}$ & Roadless forest (https://roadlessforest.eu/map.html) \\
\hline Población & $\begin{array}{l}\text { Habitantes/ } \\
\mathrm{km}^{2}\end{array}$ & $30 "$ & Densidad de población & $\begin{array}{l}\text { Gridded Population of the World v4 (http://sedac. } \\
\text { ciesin.columbia.edu/data/collection/gpw-v4) }\end{array}$ \\
\hline Puertos & $\mathrm{km}$ & $30 "$ & $\begin{array}{l}\text { Distancia lineal a puertos } \\
\text { ponderado por volumen mer- } \\
\text { cancías en } 2009\end{array}$ & $\begin{array}{l}\text { Creada a partir del listado de puertos de World Port } \\
\text { Index (https://msi.nga.mil/NGAPortal/MSI.portal) }\end{array}$ \\
\hline Cultivos & $\%$ & 5 ' & $\%$ cultivos en 2005 & $\begin{array}{l}\text { History Database of the Global Environment (HYDE v } \\
\text { 3.2) (http://themasites.pbl.nl/tridion/en/themasites/hyde/) }\end{array}$ \\
\hline Urbano & $\%$ & $30^{\prime}$ & $\%$ suelo urbano en 2005 & $\begin{array}{l}\text { History Database of the Global Environment (HYDE v } \\
\text { 3.2) (http://themasites.pbl.nl/tridion/en/themasites/hyde/) }\end{array}$ \\
\hline HII & Sin unidades & $30 "$ & Grado de antropización & $\begin{array}{l}\text { Global Human Influence Index (http://sedac.ciesin. } \\
\text { columbia.edu/data/set/wildareas-v2-human-influ- } \\
\text { ence-index-geographic) }\end{array}$ \\
\hline Carreteras & $\mathrm{km}$ & $30^{\prime \prime}$ & Distancia a carreteras & $\begin{array}{c}\text { Creada a partir de VMap0 (http://gis-lab.info/qa/ } \\
\text { vmap0-eng.html) }\end{array}$ \\
\hline PIB & $\$$ & $10^{\prime}$ & PIB por país & $\begin{array}{l}\text { Gridded Population of the World v4 (http://sedac. } \\
\text { ciesin.columbia.edu/data/collection/gpw-v4) }\end{array}$ \\
\hline
\end{tabular}

El análisis y gestión de datos geográficos se realizó mediante el software de uso libre R v 3.2.3 (R Core Team, 2017) y QGIS v 2.14.3 (QGIS Development Team, 2016).

\subsection{Variables climáticas}

Los MEN se calibran habitualmente con variables bioclimáticas que representan tendencias anuales (e.g. temperatura media anual, precipitación anual), estacionalidad (e.g. rango anual de temperatura y precipitación) y valores extremos (e.g. temperatura media del mes más frío o más cálido) promediados entre los años 1960 y 1990. En este estudio descargamos un total de 19 variables bioclimáticas a través de WorldClim-Global Climate Data v.1.4 (http://www.worldclim.org/), entre las cuales elegimos las 6 más representati- 
vas (Tabla 2): temperatura máxima (Bio5) y mínima (Bio6), precipitación máxima (Bio13) y mínima (Bio14) y estacionalidad de la precipitación (Bio15). Estas variables son utilizadas habitualmente para la calibración de MEN y guardan una relación lógica con la ecología y biología de muchas especies. Así por ejemplo, la temperatura mínima es un limitante habitual de la reproducción y crecimiento de especies, mientras que la precipitación (o su escasez) está directamente relacionada con la disponibiliad de recursos básicos y de agua corriente para el establecimiento y/o el movimiento de especies.

\subsection{Modelización de especies invasoras}

Existen varios software para calibrar y proyectar MEN entre los que destacan Maxent (Merow et al., 2013) y Biomod2 (Thuiller et al., 2014). En nuestro caso elegimos Biomod2 por dos razones: en primer lugar porque se trata de un paquete estadístico de $\mathrm{R}$ que ofrece máxima flexibilidad de calibración y capacidad de interactuar con funciones de otros paquetes estadísticos. En segundo lugar, Biomod2 permite proyectar lo que se conoce como "modelos conjunto" (ensemble models, Araújo y New, 2007), lo que consiste en calibrar un alto número de réplicas de un modelo con ligeras variaciones (ver a continuación) y calcular el promedio de todas las réplicas, lo que nos permite tener en cuenta la incertidumbre estadística inherente a los modelos.

Para calibrar Biomod2, utilizamos el listado de coordenadas donde la especie está presente y las capas ambientales que queremos utilizar como predictores. Para cada especie invasora se realizaron dos modelos: uno basado en los 6 factores climáticos ("modelo climático"), y otro que integró 6 factores climáticos y 7 humanos ("modelo integrado"). Biomod2 evalúa la calidad de los modelos en base al AUC (Area Under the ROC Curve, que debe ser $>0,8$ ) y al TSS (True Skill Statistic, que debe superar 0,7). Estos dos indicadores nos servirán para probar nuestra primera hipótesis (i.e. mayor capacidad predictiva del modelo integrado).

Como carecemos de datos independientes con los que testar la calidad de los modelos, programamos Biomod2 para dividir el listado de coordenadas en dos sets con el $50 \%$ cada uno, de modo que un set se utiliza para calibrar el modelo, y el otro para comprobar su validez (Barbet-Massin et al., 2012). Como esta división puede afectar al resultado del modelo, repetimos el proceso cinco veces (Thuiller, 2003). Por otro lado, los MEN generan por defecto 10.000 pseudo-ausencias al azar cubriendo toda el área de estudio para contrastar las características de los lugares invadidos con respecto al resto del territorio (Barbet-Massin et al., 2012). Como la selección de pseudo-ausencias también puede afectar al resultado del modelo, repetimos este proceso tres veces. Como algoritmo de modelización utilizamos cuatro de los más frecuentes para la calibración de MEN: Generalized Linear Model (GLM), Boosted Regression Model (BRM), Random forest (RF) y Generalized Additive Model (GAM) (Araújo y New, 2007).

Por tanto, para cada especie calibramos un total de 60 réplicas del modelo (resultado de 5 sets de división x 3 sets de pseudo-ausencias x 4 algoritmos). Para combinar estas réplicas en un modelo conjunto, programamos Biomod2 para descartar aquellas réplicas que obtuvieran un AUC $<0,8$ o un TSS $<0,7$, y calcular el promedio de las réplicas 
restantes, ponderadas por su TSS (es decir, una réplica con TSS de 0.9 pesará más en el resultado final que una con TSS de 0,7).

Una vez calibrados, los MEN se pueden proyectar en el espacio o el tiempo. En este caso, al tratarse de especies invasoras de interés europeo, los proyectamos en Europa. El resultado es un mapa de idoneidad con valores que van de 0 a 1000 en función de la similitud entre cada pixel de 5' y las zonas actualmente ocupadas por la especie. Adicionalmente, el programa nos ofrece un valor umbral a partir del cual podemos considerar que la probabilidad de establecimiento es mayor que la de ausencia, que no necesariamente tiene que ser 500. En nuestro caso elegimos el umbral conocido como "maximum training sensitivity and especificity" (maxTSS), uno de los más utilizados para modelizar especies invasoras (Barbet-Massin et al., 2012). Una vez utilizado este umbral para generar un mapa binario (presencia/ausencia), calculamos el área total predicha como idónea para la especie de acuerdo al modelo climático y el humano. Esto nos permitió testar nuestra segunda hipótesis: la presión de propágulos incrementa el área susceptible a la invasión, especialmente en regiones antropizadas como la europea. Además, solapamos los mapas binarios climático e integrado para localizar las zonas de solape y de discrepancia entre ambas opciones.

Finalmente, sumamos los mapas binarios de presencia/ausencia correspondientes a las especies modelizadas para generar un mapa de riqueza, con valores de 0 a 57 , que nos facilite identificar las zonas de mayor riesgo de invasión (hotspots) en Europa de acuerdo a los modelos climático e integrado. Además, calculamos la diferencia entre el mapa de riqueza integrado y el climático. Para confirmar nuestra segunda y tercera hipótesis, los valores positivos (i.e. mayor idoneidad según el modelo integrado) deberían corresponder a las zonas más antropizadas en Europa, como las zonas costeras, grandes ciudades y alrededores del canal de la Mancha (Inglaterra, norte de Francia, Bélgica y Países Bajos). Por su parte, los valores negativos (i.e. mayor idoneidad según el modelo climático) deberían localizarse en las zonas más prístinas: Península noruega, norte de Escocia, países del Este y zonas de alta montaña (Pirineos, Alpes). El mapa de accesibilidad disponible en la Figura S1 aporta una indicación rápida de las zonas más y menos desarrolladas en Europa.

\section{Resultados}

\subsection{Principales vectores de introducción}

Cada uno de los grupos taxonómicos evaluados está dominado por un tipo de vector de introducción diferente, de un total de nueve grandes vectores identificados (Tabla 1). Así por ejemplo, el vector más importante para la introducción de especies invasoras de agua dulce en Europa es el transporte (para 10 de las 13 especies consideradas), seguido de la pesca (6 especies), la acuicultura (5 especies) y los canales que conectan cuencas hidrográficas (5 especies). Los 11 invertebrados terrestres tienen como principal vector el transporte y comercio (como polizones o contaminantes de la mercancía), fundamentalmente marítimo pero también aéreo y rodado. Las plantas se introducen mayoritariamente a través del comercio ornamental, la explotación hortícola o forestal ( 15 de 18 especies evaluadas), y también suelen utilizarse para la restauración de suelos y el control de la erosión (7 especies). Finalmente, en el caso de los 
vertebrados, son frecuentemente introducidos de forma deliberada para la caza, cría y comercio de pieles (11 de 15 especies evaluadas), seguido por el comercio de mascotas (5 especies).

\subsection{Los modelos ecológicos de nicho: ¿es mejor el modelo climático o el integrado?}

\subsubsection{Diferencias en cuanto a los estadísticos del modelo}

Los modelos climáticos obtuvieron valores de precisión generalmente altos (TSS > 0,7 y AUC > 0,8). Aunque los modelos integrados ofrecen valores ligeramente superiores, la diferencia no es significativa (ANOVA con TSS: $\mathrm{df}=1,112$; F-valor $=1,73 ; \mathrm{P}>$ 0,05, valores similares con AUC). Los resultados por cada especie se pueden consultar en la Tabla S2 y por grupos taxonómicos en la Tabla 3.

Tabla 3. Resumen de los resultados del modelo con variables climáticas únicamente, y del modelo que integra variables climáticas y humanas. TSS: True Skill Statistics, medida de la calidad del modelo. Se incluye el porcentaje de contribución al modelo de la(s) variable(s) más importantes. Bio6: Temperatura mínima mes más frío. Bio5: Temperatura máxima mes más cálido. Puertos: distancia lineal a puertos comerciales. Acces.: tiempo de viaje a la gran ciudad (> 50.000 habitantes) más cercana.

\begin{tabular}{|l|c|c|c|c|c|c|c|c|}
\hline & \multicolumn{3}{|c|}{ Modelo clima } & \multicolumn{5}{c|}{ Modelo integrado } \\
\hline Grupo & TSS & Bio6 & Bio5 & TSS & Bioclim & Puertos & PIB & Acces. \\
\hline Acuáticas & $0,87 \pm 0,3$ & $0,55 \pm 0,09$ & $0,27 \pm 0,08$ & $0,88 \pm 0,03$ & $0,56 \pm 0,14$ & $0,12 \pm 0,11$ & $0,08 \pm 0,10$ & $0,08 \pm 0,09$ \\
\hline Invertebrados & $0,79 \pm 0,2$ & $0,64 \pm 0,06$ & $0,33 \pm 0,07$ & $0,83 \pm 0,03$ & $0,55 \pm 0,12$ & $0,10 \pm 0,09$ & $0,11 \pm 0,08$ & $0,07 \pm 0,09$ \\
\hline Vertebrados & $0,77 \pm 0,6$ & $0,53 \pm 0,07$ & $0,22 \pm 0,08$ & $0,82 \pm 0,04$ & $0,52 \pm 0,08$ & $0,12 \pm 0,07$ & $0,12 \pm 0,06$ & $0,08 \pm 0,06$ \\
\hline Plantas & $0,88 \pm 0,2$ & $0,60 \pm 0,05$ & $0,32 \pm 0,05$ & $0,88 \pm 0,02$ & $0,60 \pm 0,10$ & $0,10 \pm 0,07$ & $0,10 \pm 0,06$ & $0,05 \pm 0,07$ \\
\hline
\end{tabular}

*Bioclim: suma de las 6 variables bioclimáticas

La variable con mayor peso en el modelo climático es la temperatura mínima del mes más frío (Bio6), responsable de entre el 30 y $90 \%$ de la distribución de las especies (media $58 \%$ ). Esta variable se correlaciona positivamente con la riqueza potencial de especies invasoras en Europa $(r=0,65, \mathrm{p}<0,001)$, lo que significa que a mayor temperatura mínima, mayor número de especies invasoras pueden establecerse. La temperatura mínima sigue siendo la variable más relevante en el modelo integrado (entre 10 y $66 \%$ de contribución al modelo, media 32\%), seguida de la distancia a puertos, el PIB y la accesibilidad (Tabla 3). La accesibilidad se correlaciona negativamente con la riqueza de especies invasoras $(r=$ $-0,54, \mathrm{p}<0,001)$. Dado que la accesibilidad se mide como "tiempo de viaje a la ciudad más cercana", podemos interpretar que a mayor tiempo de viaje (zonas más aisladas), menor probabilidad de invasión. De hecho, sería más correcto llamar a esta variable "aislamiento" en lugar de accesibilidad, pero hemos decidido utilizar su denominación original.

\subsubsection{Diferencias en cuanto a superficie idónea para la invasión}

La mayor diferencia entre los modelos climático e integrado la encontramos en las proyecciones espaciales. Aunque la diferencia entre modelos de nuevo no es significativa (ANOVA con área total considerada idónea: df $=1,112$; F-valor $=0,012 ; \mathrm{P}>0,05$ ), el modelo integrado tiende a predecir una mayor superficie susceptible de invasión en el caso 
de los vertebrados, y menor en el caso de invertebrados y organismos acuáticos (Tabla 4). No obstante, los datos difieren enormemente entre especies, tal y como puede apreciarse en la Figura 2. Así por ejemplo, encontramos vertebrados tanto entre las especies que más superficie ganan en el modelo integrado (como el visón americano, el galápago de Florida y la malvasía canela) como entre las que más pierden (la rata parda y el ganso de Canadá). De hecho, no apreciamos diferencias significativas en cuanto al área predicha como idónea para los distintos grupos taxonómicos (ANOVA: df =3,110; F-valor =0,25, P >0,05).

Tabla 4. Superficie total predicha por los modelos climáticos e integrados como vulnerable para el establecimiento de especies invasoras. Los datos corresponden al sumatorio de todas las especies en cada grupo, en millones de hectáreas (Mha). Diferencia: resultado de restar la superficie según el modelo integrado de la del modelo climático. \% cambio: porcentaje de aumento o disminución respecto a la superficie estimada por el modelo climático.

\begin{tabular}{|l|c|c|c|c|}
\hline & Modelo Clima & Modelo Integrado & Diferencia & \% cambio \\
\hline Acuáticas & 3560,00 & 3553,60 & $-6,40$ & $-1,16$ \\
\hline Invertebrados & 3045,60 & 2933,70 & $-111,90$ & $-2,08$ \\
\hline Vertebrados & 4081,00 & 4204,00 & 123,00 & 14,24 \\
\hline Plantas & 4503,60 & 4633,00 & 129,40 & 11,82 \\
\hline
\end{tabular}

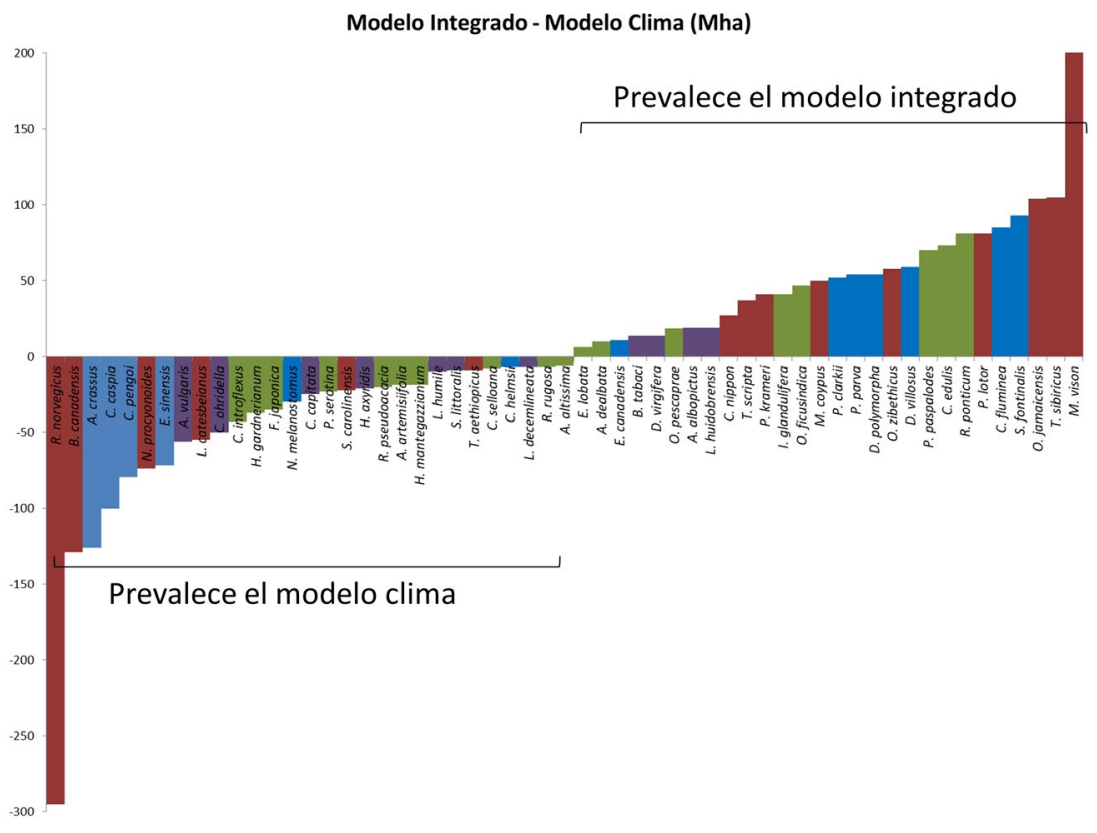

Figura 2. Cambio en la superficie total predicha como idónea para cada especie entre el modelo climático y el modelo integrado. Valores positivos indican un aumento de superficie idónea en el modelo integrado y viceversa. Datos en millones de hectáreas (Mha). Los colores de las barras representan el grupo taxonómico de la especie. Rojo: vertebrados terrestres, azul: organismos de agua dulce, morado: invertebrados terrestres, y verde: plantas. 
Además de un cambio neto en la superficie total predicha como idónea, también podemos apreciar un cambio espacial en las zonas resaltadas por cada tipo de modelo (ejemplos en Fig. 3). Observamos que en la mayoría de los casos hay un solape importante entre los dos modelos (zonas en color verde), si bien el modelo integrado tiende a expandir el área de establecimiento potencial hacia zonas antropizadas (zonas en color rojo). Es el caso del visón americano ( $M$. vison, Fig. 3C), cuya distribución aparece limitada en el sur de la Península Ibérica según el modelo climático, mientras que el modelo integrado sugiere un potencial de expansión muy superior. Lo mismo ocurre con el mapache (P. lotor, Fig. 3D) y la almeja asiática (C. fluminea, Fig. 3E). Por el contrario, especies como el jengibre hawaiano (H. gardnerianum, Fig. 3A) o el cangrejo chino (E. sinensis, Fig. 3B) muestran zonas idóneas climáticamente (zonas en color azul) donde la falta de propágulos podría limitar la expansión de la especie.

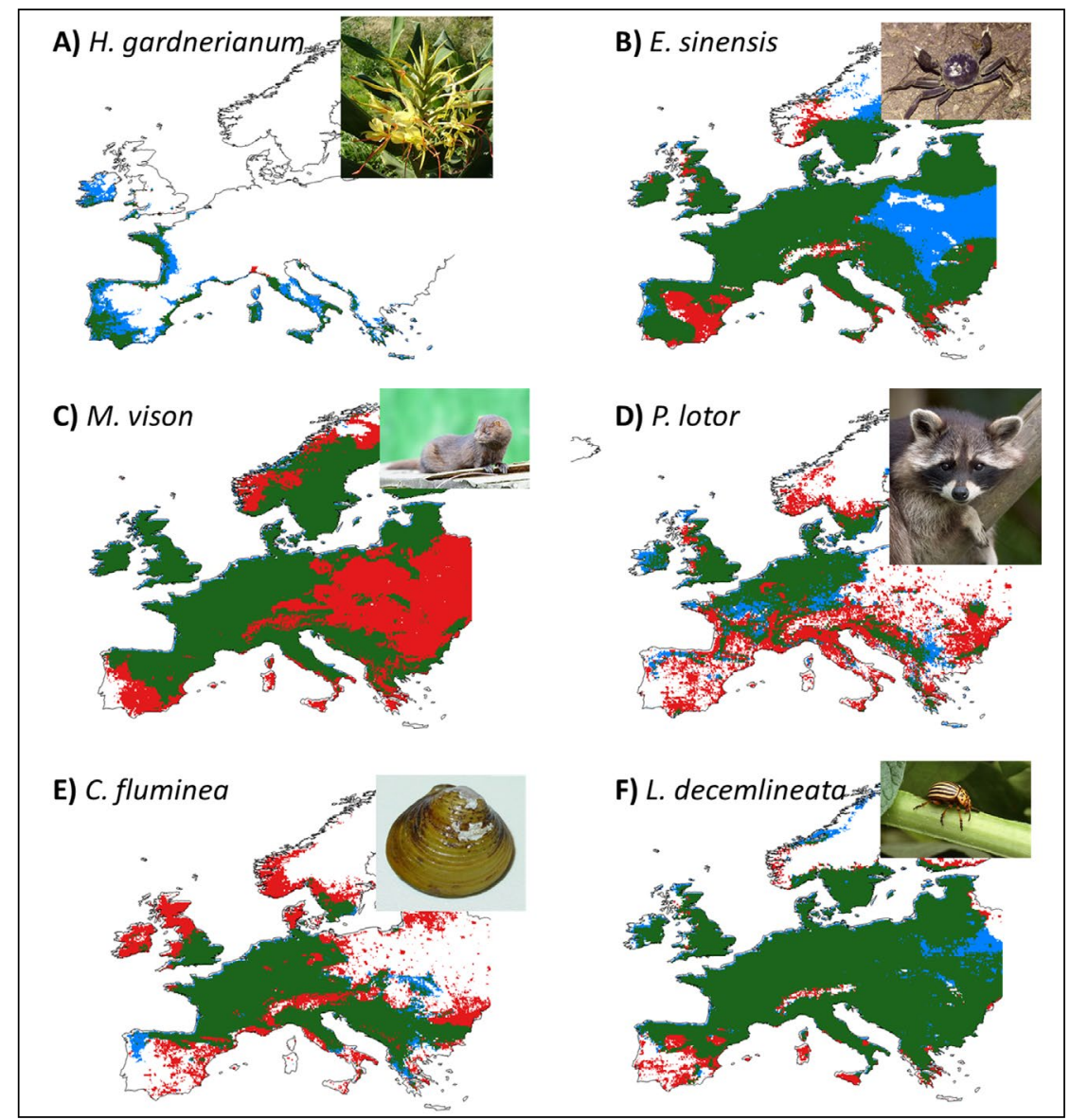

Figura 3. Comparación entre las predicciones del modelo climático (azul) y el modelo integrado (rojo). Las zonas en color verde son clasificadas como idóneas para la especie por ambos modelos. A: Hedychium gardnerianum, jengibre blanco. B: Eriocheir sinensis, cangrejo chino.

C: Mustela (Neovison) vison, visón americano. D: Procyon lotor, mapache. E: Corbicula fluminea, almeja asiática. F: Leptinotarsa decemlineata, escarabajo de la patata. 


\subsubsection{Cambio en cuanto a la riqueza de especies invasoras}

Si sumamos todos los mapas, obtenemos un mapa de riqueza con valores de 0 a 57 en función del número de especies que encontrarían condiciones idóneas para establecerse (Fig. 4). La media del mapa climático es de $23,7(\mathrm{SD}=15,22$, máximo $=53$ especies, Fig. 4A), mientras que en el caso del mapa integrado, la media es de 25,6 $(\mathrm{SD}=15,8$, máximo $=55$ especies, Fig. 4B). En la Península Ibérica, el modelo climático señala la franja norte peninsular como la más proclive a las invasiones biológicas (Fig. 4C), mientras que en el integrado aumentan los valores de riesgo en el levante, valle del Ebro y alrededores de grandes ciudades (Fig. 4D). Finalmente, el grado de influencia humana es significativamente superior en aquellas zonas europeas en las que el modelo integrado aumenta la probabilidad de invasión (valores positivos en rojo en la Fig. 4B), que aquellas en las que disminuye (valores negativos en azul en la Fig. 4B) (ANOVA con idoneidad: $\mathrm{df}=1,112$; F-valor $=154,5 ; \mathrm{P}<0,001$ ).

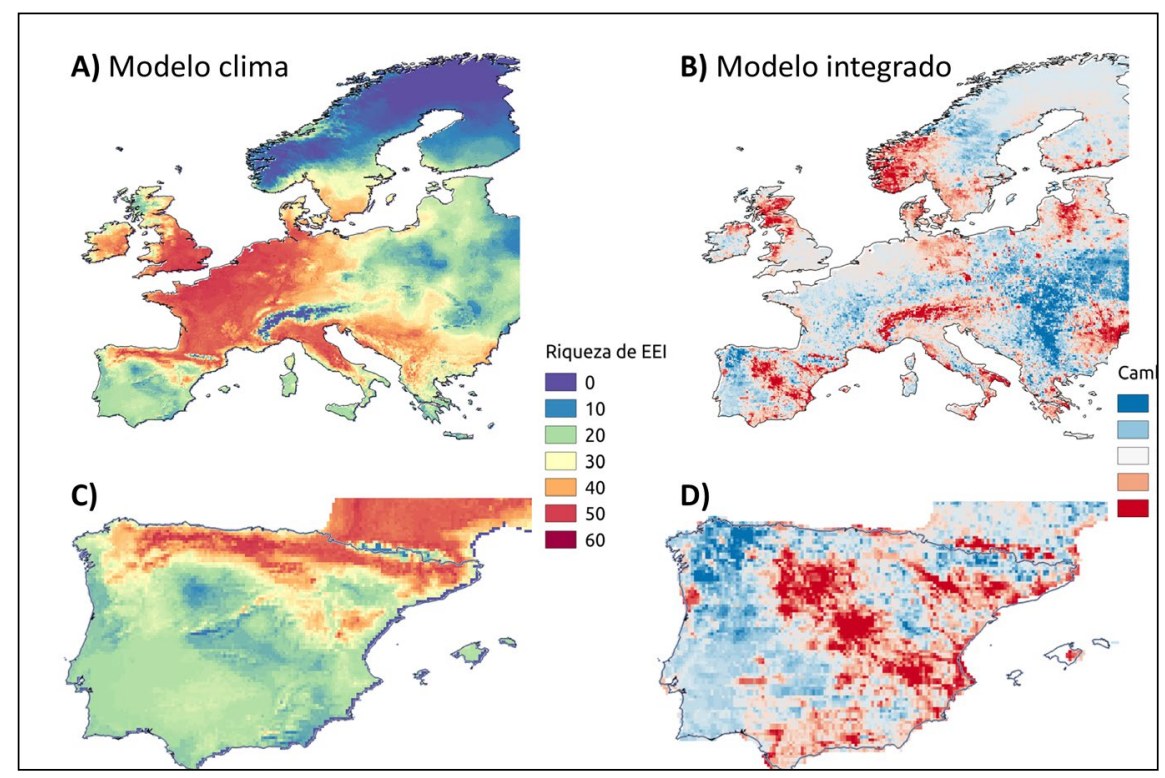

Figura 4. Riqueza potencial de especies invasoras en Europa en función al modelo climático y al modelo integrado. Los valores del modelo clima $(A$ y $C)$ reflejan el número total de especies exóticas invasoras (EEI) que podrían encontrar condiciones idóneas para su establecimiento. Los valores del mapa integrado $(B$ y $D$ ) representan el cambio (positivo en rojo, negativo en azul) en el número total de EEI del modelo clima.

\section{Discusión}

\subsection{Principales vectores de introducción}

Las especies invasoras llegan a Europa de diversas formas, de hecho la mayoría cuentan con varios vectores de introducción principales y secundarios. Destaca claramente el transporte, vector principal de 25 de las 57 especies evaluadas, seguido del cultivo ornamental (17 
especies) y el comercio (16 especies). Sin embargo podemos apreciar diferencias importantes entre grupos taxonómicos. La mayoría de las especies invasoras de agua dulce han llegado desde otros continentes a través del transporte marítimo, bien adheridas al casco de los barcos o en los tanques de agua de lastre (Briski et al., 2012; Banks et al., 2014). Una vez introducidas, el transporte por carretera facilita la dispersión secundaria en el caso de especies capaces de soportar la desecación, como contaminantes de aperos de pesca o adheridos a pequeñas embarcaciones remolcadas (e.g. Alonso y Castro-Díez, 2012). Las plantas son introducidas mayoritariamente a través de la horticultura, si bien en algunos casos se trata de malas hierbas o contaminantes de semillas introducidas de forma accidental (Lambdon et al., 2008). Por el contrario, los invertebrados terrestres tales como pulgones, hormigas y polillas, suelen llegar como contaminantes de mercancías, sobre todo productos agrícolas, forestales u ornamentales (Roques et al., 2009). La excepción es la mariquita asiática, Harmonia axyridis, que fue introducida para el control biológico de coccinélidos, y acabó expandiéndose fuera de control (Roy y Wajnberg, 2008). La gran mayoría de los vertebrados terrestres han sido introducidos intencionadamente para la caza (e.g. ciervo japonés), comercio de pieles (e.g. visón americano, coipú y rata almizclera), o como mascotas (e.g. mapache, cotorra de Kramer, ardilla gris, ardilla de Siberia y galápago de Florida) (Genovesi et al., 2009; Kark et al., 2009).

\section{2. ¿Obtienen los modelos integrados una mayor calidad y capacidad predictiva que modelos basados únicamente en variables climáticas?}

No, a pesar de que los modelos integrados ofrecen valores de precisión (AUC y TSS) ligeramente superiores a los modelos basados en variables climáticas, la diferencia no es significativa. Por tanto, no podemos confirmar, al menos estadísticamente, esta hipótesis.

No obstante, hay que tener en cuenta que los modelos de distribución de especies tienden a proporcionar valores de AUC cada vez más altos a medida que aumentamos la extensión de estudio (Lobo et al., 2008). El AUC es una medida de discriminación que mide la probabilidad de asignar correctamente una presencia, tarea que es cada vez más sencilla a medida que aumentamos la extensión del estudio y cubrimos un gradiente de condiciones ambientales mayor. Esta "inflación" del AUC se puede ver claramente en nuestro estudio, con valores que oscilan entre 0,89 y 0,99 para los modelos climático e integrado, un rango tan estrecho que difícilmente encontraremos diferencias significativas. Es por ello que añadimos como estadístico de contraste el TSS, menos susceptible a la inflación (o al menos no hay estudios que lo hayan comprobado), aunque tampoco encontramos diferencias significativas. Nuestros resultados son coherentes con investigaciones previas (Bucklin et al., 2014; Zhu et al., 2016), donde tan solo observaron un ligero aumento de capacidad predictiva de los modelos integrados.

Dicho esto, sorprendentemente la influencia humana fue capaz de explicar entre un $39 \%$ (plantas) y $48 \%$ (vertebrados) de la distribución global de especies invasoras, en línea con lo que hemos observado en estudios previos (Gallardo et al., 2015). Entre las variables humanas destacan la distancia a puertos (sobre todo para organismos acuáticos), el PIB (vertebrados), y las carreteras (plantas); los cuales guardan una estrecha relación con los vectores principales de introducción de cada grupo de especies identificados en la Tabla 1. 
La influencia del hombre sobre los nuevos patrones biogeográficos del Antropoceno está ganando atención rápidamente por sus aplicaciones prácticas, sobre todo en cuanto a la prevención de riesgos biológicos. No obstante esta relación es compleja y probablemente varíe con la escala de estudio, por lo que todavía necesitamos más estudios que ayuden a identificar qué variables humanas son importantes en función de la especie y el área de estudio.

\section{3. ¿Subestiman los modelos climáticos el potencial de invasión en zonas con elevada influencia humana?}

Sí, observamos un aumento general del 8\% en el área total susceptible de ser invadida cuando tenemos en cuenta variables humanas; una cifra nada desdeñable pero inferior a la esperada, y mucho más baja que el $20 \%$ observado en estudios sobre organismos acuáticos (Gallardo y Aldridge, 2013). Los resultados varían mucho entre especies y el aumento en superficie susceptible a la invasión es muy superior en el caso de vertebrados (14\%) y plantas (12\%), que en el de organismos de agua dulce (1\%) e invertebrados (2\%). Incluso en casos en los que la variación neta del área total no parece importante, los mapas de solape demuestran cómo los modelos integrados generalmente expanden el área vulnerable a la invasión hacia zonas muy antropizadas. Esto se aprecia claramente en el caso de la Península Ibérica, donde los modelos integrados incrementan el riesgo de invasión en el centro peninsular, valle del Ebro y costa del Levante (colores rojizos, Fig. 4D), caracterizados por una fuerte presión antrópica. Es más, el grado de influencia humana es significativamente superior en las zonas de mayor prevalencia del modelo integrado, lo que parece confirmar nuestra segunda hipótesis.

Estudios recientes confirman la capacidad de las especies invasoras para establecer poblaciones en áreas con baja idoneidad climática pero donde la influencia humana permite su supervivencia bien mediante un aporte continuo de propágulos, bien a través de micro-refugios climáticos, o una combinación de ambas (Roura-Pascual et al., 2011; Beans et al., 2012). También se ha demostrado en la literatura científica una relación directa entre la riqueza de especies invasoras y el grado de antropización, que en el caso de Europa son máximos en los países alrededor del Canal de la Mancha (Inglaterra, Francia, Bélgica y Países Bajos) (Gallardo et al., 2015; Bellard et al., 2016).

Las invasiones biológicas pueden ser vistas como un proceso altamente estocástico, y por tanto difícil de prever. Pero los modelos de distribución de especies han demostrado repetidamente su capacidad para anticipar, al menos a escala regional, tanto las zonas de establecimiento inicial como posterior dispersión (Gallardo et al., 2013; Barbet-Massin et al., 2018). Una de las principales ventajas de esta herramienta es la facilidad y rapidez de calibración que ofrece, dado que tan solo requiere datos georreferenciados de presencia de la especie y una serie de predictores básicos disponibles públicamente, ofreciendo un escenario base de invasión potencial con el que poder trabajar. No obstante, hemos de recordar que los mapas de idoneidad reflejan la probabilidad de que una especie se establezca con éxito si es introducida, lo que no implica necesariamente que esto ocurra, ya que hay otra serie de factores bióticos (e.g. efectos cuello de botella en la población invasora), abióticos (e.g. presencia de micro-refugios o de factores limitantes desconocidos) y humanos (e.g. perturbación del hábitat) determinantes. Si bien la 
integración de factores relacionados con la influencia humana mejora las predicciones espaciales del modelo, el grado de incertidumbre de una proyección sigue siendo elevado y ha de ser interpretado con cautela.

\section{4. ¿Sobreestiman los modelos climáticos el potencial invasor en regiones relativamen- te prístinas y alejadas de la influencia humana?}

En este estudio no hemos podido confirmar con claridad esta hipótesis, lo que puede deberse en gran medida a la falta de zonas prístinas en un continente muy humanizado (Fig. S1). Efectivamente, podemos observar una mayor prevalencia del modelo climático en las zonas más aisladas de Europa, tales como el norte de la Península Escandinava y los países del este de Europa (colores azulados, Fig. 4B). Sin embargo, otras zonas a priori aisladas y poco humanizadas contradicen nuestra hipótesis, como es el caso del norte de Escocia, Suecia y la zona de los Alpes donde el modelo integrado prevalece a pesar de que podemos suponer una presión de propágulos más bien baja.

En el caso de la Península Ibérica, las zonas de menor influencia humana y en las que prevalece el modelo climático corresponden al interior de Portugal, Galicia, interior de Extremadura, la zona de Soria-Teruel y los Pirineos (colores azulados en la Fig. 4D). En esta línea, en un reciente estudio comprobamos cómo los Parques Nacionales más aislados, en algunos casos declarados a principios del siglo XX, no registran ninguna o muy pocas especies invasoras, mientras que los espacios protegidos más accesibles, a menudo declarados recientemente como espacios Red Natura 2000, contienen un elevado número de invasores (Gallardo et al., 2017).

Más allá de estas apreciaciones espaciales, no hemos podido comprobar estadísticamente esta hipótesis.

\section{Conclusiones}

La principal conclusión de este estudio es que el clima es importante, pero no lo suficiente desde un punto de vista explicativo a la hora de anticipar futuras invasiones. Para evitar subestimar el potencial de invasión, debemos integrar de manera rutinaria información sobre la influencia humana en cualquier modelo predictivo. Variables humanas relacionadas con el transporte (tales como accesibilidad, distancia a puertos o carreteras), el desarrollo económico (PIB) y grado de antropización de los ecosistemas (Human Influence Index), mejoran las predicciones espaciales de los modelos, pero son genéricas y no cubren todos los vectores de introducción conocidos. Teniendo en cuenta la disponibilidad creciente de todo tipo de datos, urge sintetizar y estandarizar la información disponible para crear mapas a escala global y con una resolución suficiente (10 arco-minutos o más) que permita cubrir importantes vectores de distribución de especies invasoras, sobre todo los relacionados con el comercio de distintos tipos de mercancías, la acuicultura y la pesca. Por ejemplo, en este estudio hemos utilizado mapas vectoriales de carreteras y de puertos para crear un mapa de distancias que nos permita considerar la influencia de los vectores de transporte. Aunque estas nuevas variables han demostrado 
ser útiles para investigar la distribución a gran escala de especies invasoras, sería interesante poder incorporar información acerca del volumen de tráfico que se mueve a través de cada carretera o puerto para mejorar las predicciones.

Los resultados de este estudio ofrecen información básica acerca de las zonas más y menos susceptibles a la invasión, a partir de las cuales se pueden diseñar estrategias de prevención, respuesta inmediata y erradicación de especies invasoras efectivas, tal y como recomienda la Regulación 1143/2014 sobre especies invasoras de la Unión Europea.

\section{Agradecimientos}

La investigación aquí presentada ha sido financiada por el Plan Nacional de I+D+i, Proyectos orientados a los retos de la sociedad (CGL-2014-55145-R) y por el projecto InvasiBES (PCI2018-092939) financiado por la ERA-Net BiodivERsA-Belmont Forum. BG es investigadora postdoctoral a cargo del programa Juan de la Cierva (JCl-2012-11908). Este estudio ha sido elaborado a partir del Trabajo Fin de Master de L.V. Los autores agradecen el apoyo técnico de Manuel Pizarro Gavilán.

\section{Referencias}

Alonso, A., Castro-Díez, P. 2012. Tolerance to air exposure of the new zealand mudsnail Potamopyrgus antipodarum (Hydrobiidae, Mollusca) as a prerequisite to survival in overland translocations. NeoBiota 14, 67-74. https://doi.org/10.3897/neobiota.14.3140.

Araújo, M.B., New, M. 2007. Ensemble forecasting of species distributions. Trends in Ecology \& Evolution 22, 42-47. https://doi.org/10.1016/j.tree.2006.09.010.

Banks, N.C., Paini, D.R., Bayliss, K.L., Hodda, M. 2014. The role of global trade and transport network topology in the human-mediated dispersal of alien species. Ecology Letters 18, 188199. https://doi.org/10.1111/ele.12397.

Barbet-Massin, M., Jiguet, F., Albert, C.H., Thuiller, W. 2012. Selecting pseudo-absences for species distribution models: How, where and how many? Methods in Ecology and Evolution 3, 327-338. https://doi.org/10.1111/j.2041-210X.2011.00172.x.

Barbet-Massin, M., Rome, Q., Villemant, C., Courchamp, F. 2018. Can species distribution models really predict the expansion of invasive species? PLOS ONE 13, e0193085. https://doi. org/10.1371/journal.pone.0193085.

Beans, C.M., Kilkenny, F.F., Galloway, L.F. 2012. Climate suitability and human influences combined explain the range expansion of an invasive horticultural plant. Biological Invasions 14, 2067-2078. https://doi.org/10.1007/s10530-012-0214-0.

Bellard, C., Leroy, B., Thuiller, W., Rysman, J.F., Courchamp, F. 2016. Major drivers of invasion risks throughout the world. Ecosphere 7, e01241. https://doi.org/10.1002/ecs2.1241.

Briski, E., Ghabooli, S., Bailey, S., MacIsaac, H. 2012. Invasion risk posed by macroinvertebrates transported in ships' ballast tanks. Biological Invasions 14, 1843-1850. https://doi. org/10.1007/s10530-012-0194-0.

Broennimann, O., Guisan, A. 2008. Predicting current and future biological invasions: Both native and invaded ranges matter. Biology Letters 4, 585-589. https://doi.org/10.1098/ rsbl.2008.0254.

Bucklin, D.N., Basille, M., Benscoter, A.M., Brandt, L.A., Mazzotti, FJ., Romañach, S.S., Speroterra, C., Watling, J.I. 2014. Comparing species distribution models constructed with different subsets of environmental predictors. Diversity and Distributions 21, 23-35. https://doi.org/10.1111/ddi.12247. 
Callaway, R.M., Ridenour, W.M. 2004. Novel weapons: Invasive success and the evolution of increased competitive ability. Frontiers in Ecology and the Environment 2, 436-443. https:// doi.org/10.1890/1540-9295(2004)002[0436:NWISAT]2.0.CO;2.

Capinha, C., Essl, F., Seebens, H., Moser, D., Pereira, H.M. 2015. The dispersal of alien species redefines biogeography in the Anthropocene. Science 348, 1248-1251. https://doi.org/10.1126/ science.aaa8913.

Estrada, A., Morales-Castilla, I., Caplat, P., Early, R. 2016. Usefulness of species traits in predicting range shifts. Trends in Ecology \& Evolution 31, 190-203. https://doi.org/10.1016/j. tree.2015.12.014.

Gallardo, B., Aldridge, D.C. 2013. The 'dirty dozen': socio-economic factors amplify the invasion potential of 12 high risk aquatic invasive species in great britain and ireland. Journal of Applied Ecology 50, 757-766. https://doi.org/10.1111/1365-2664.12079.

Gallardo, B., Zu Ermgassen, P.S.E., Aldridge, D.C. 2013. Invasion ratcheting in the zebra mussel (Dreissena polymorpha) and the ability of native and invaded ranges to predict its global distribution. Journal of Biogeography 40, 2274-2284. https://doi.org/10.1111/ jbi.12170.

Gallardo, B., Zieritz, A., Aldridge, D.C. 2015. The importance of the human footprint in shaping the global distribution of terrestrial, freshwater and marine invaders. PLOS ONE 10, e0125801. https://doi.org/10.1371/journal.pone.0125801.

Gallardo, B., Aldridge, D.C., González-Moreno, P., Pergl, J., Pizarro, M., Pyšek, P., Thuiller, W., Yesson, C., Vilà, M. 2017. Protected areas offer refuge from invasive species spreading under climate change. Global Change Biology 23, 5331-5343. https://doi.org/10.1111/ gcb.13798.

Gallardo, B., Bogan, A.E., Harun, S., Jainih, L., Lopes-Lima, M., Pizarro, M., Rahim, K.A., Sousa, R., Virdis, S.G.P., Zieritz, A. 2018. Current and future effects of global change on a hotspot's freshwater diversity. Science of The Total Environment 635, 750-760. https://doi. org/10.1016/j.scitotenv .2018.04.056.

Genovesi, P., Bacher, S., Kobelt, M., Pascal, M.,Scalera, R. 2009. Alien mammals of Europe. Handbook of alien species in europe, pp. 119-128. Springer.

Guisan, A.,Thuiller, W. 2005. Predicting species distribution: offering more than simple habitat models. Ecology Letters 8, 993-1009. https://doi.org/10.1111/j.1461-0248.2005.00792.x.

Hampton, S.E., Strasser, C.A., Tewksbury, J.J., Gram, W.K., Budden, A.E., Batcheller, A.L., Duke, C.S., Porter, J.H. 2013. Big data and the future of ecology. Frontiers in Ecology and the Environment 11, 156-162. https://doi.org/10.1890/120103.

Hijmans, R.J., Graham, C.H. 2006. The ability of climate envelope models to predict the effect of climate change on species distributions. Global Change Biology 12, 2272-2281. https://doi. org/10.1111/j.1365-2486.2006.01256.x.

Kark, S., Solarz, W., Chiron, F., Clergeau, P., Shirley, S. 2009. Alien birds, amphibians and reptiles of europe. Handbook of alien species in europe. Springer. pp. 105-118.

Katsanevakis, S., Bogucarskis, K., Gatto, F., Vandekerkhove, J., Deriu, I., Cardoso, A.C. 2012. Building the european alien species information network (EASIN): a novel approach for the exploration of distributed alien species data. BioInvasions Record 1, 235-245. http://doi. org/10.3391/bir.2012.1.4.01.

Lambdon, P.W., Pyšek, P., Basnou, C., Hejda, M., Arianoutsou, M., Essl, F., Jarošík, V., Pergl, J., Winter, M., Anastasiu, P. 2008. Alien flora of europe: species diversity, temporal trends, geographical patterns and research needs. Preslia 80, 101-149.

Lobo, J.M., Jimenez-Valverde, A., Real, R. 2008. AUC: A misleading measure of the performance of predictive distribution models. Global Ecology and Biogeography 17, 145-151. https:// doi.org/10.1111/j.1466-8238.2007.00358.x. 
Lockwood, J.L., Cassey, P., Blackburn, T. 2005. The role of propagule pressure in explaining species invasions. Trends in Ecology \& Evolution 20, 223-228. https://doi.org/10.1016/j. tree.2005.02.004.

Merow, C., Smith, M.J., Silander, J.A. 2013. A practical guide to Maxent for modeling species' distributions: what it does, and why inputs and settings matter. Ecography 36, 1058-1069. https://doi.org/10.1111/j.1600-0587.2013.07872.x.

Olden, J.D., Douglas, M.E., Douglas, M.R. 2005. The human dimensions of biotic homogenization. Conservation Biology 19, 2036-2038. https://doi.org/10.1111/j.1523-1739.2005.00288.x.

Olden, J.D., LeRoy Poff, N., Douglas, M.R., Douglas, M.E., Fausch, K.D. 2004. Ecological and evolutionary consequences of biotic homogenization. Trends in Ecology \& Evolution 19, 1824. https://doi.org/10.1016/j.tree.2003.09.010.

QGIS Development Team (2016) QGIS geographic information system. Open source geospatial foundation project.

R Core Team (2017) $R$ : A language and environment for statistical computing. R Foundation for Statistical Computing

Roques, A., Rabitsch, W., Rasplus, J.-Y., Lopez-Vaamonde, C., Nentwig, W., Kenis, M. 2009. Alien terrestrial invertebrates of europe. Handbook of alien species in europe. Springer, pp. 63-79.

Roura-Pascual, N., Hui, C., Ikeda, T., Leday, G., Richardson, D.M., Carpintero, S., Espadaler, X., Gómez, C., Guénard, B., Hartley, S., Krushelnycky, P., Lester, P.J., McGeoch, M.A., Menke, S.B., Pedersen, J.S., Pitt, J.P.W., Reyes, J., Sanders, N.J., Suarez, A.V., Touyama, Y., Ward, D., Ward, P.S., Worner, S.P. 2011. Relative roles of climatic suitability and anthropogenic influence in determining the pattern of spread in a global invader. Proceedings of the National Academy of Sciences 108, 220-225. https://doi.org/10.1073/pnas.1011723108.

Roy, H., Wajnberg, E. 2008. From biological control to invasion: The ladybird harmonia axyridis as a model species. BioControl 53,1-4. https://doi.org/10.1007/s10526-007-9127-8.

Sanderson, E.W., Jaiteh, M., Levy, M.A., Redford, K.H., Wannebo, A.V., Woolmer, G. 2002. The human footprint and the last of the wild. Bioscience 52, 891-904. https://doi.org/10.1641/0006-3568(2002)052[0891:thfatl]2.0.co;2.

Seebens, H., Blackburn, T.M., Dyer, E.E., Genovesi, P., Hulme, P.E., Jeschke, J.M., Pagad, S., Pyšek, P., Winter, M., Arianoutsou, M., Bacher, S., Blasius, B., Brundu, G., Capinha, C., Celesti-Grapow, L., Dawson, W., Dullinger, S., Fuentes, N., Jäger, H., Kartesz, J., Kenis, M., Kreft, H., Kühn, I., Lenzner, B., Liebhold, A., Mosena, A., Moser, D., Nishino, M., Pearman, D., Pergl, J., Rabitsch, W., Rojas-Sandoval, J., Roques, A., Rorke, S., Rossinelli, S., Roy, H.E., Scalera, R., Schindler, S., Štajerová, K., Tokarska-Guzik, B., van Kleunen, M., Walker, K., Weigelt, P., Yamanaka, T., Essl, F. 2017. No saturation in the accumulation of alien species worldwide. Nature Communications 8, 14435. https://doi.org/10.1038/ncomms14435.

Seebens, H., Blackburn, T.M., Dyer, E.E., Genovesi, P., Hulme, P.E., Jeschke, J.M., Pagad, S., Pyšek, P., van Kleunen, M., Winter, M., Ansong, M., Arianoutsou, M., Bacher, S., Blasius, B., Brockerhoff, E.G., Brundu, G., Capinha, C., Causton, C.E., Celesti-Grapow, L., Dawson, W., Dullinger, S., Economo, E.P., Fuentes, N., Guénard, B., Jäger, H., Kartesz, J., Kenis, M., Kühn, I., Lenzner, B., Liebhold, A.M., Mosena, A., Moser, D., Nentwig, W., Nishino, M., Pearman, D., Pergl, J., Rabitsch, W., Rojas-Sandoval, J., Roques, A., Rorke, S., Rossinelli, S., Roy, H.E., Scalera, R., Schindler, S., Štajerová, K., Tokarska-Guzik, B., Walker, K., Ward, D.F., Yamanaka, T., Essl, F. 2018. Global rise in emerging alien species results from increased accessibility of new source pools. Proceedings of the National Academy of Sciences. https://doi.org/10.1073/pnas.1719429115.

Soberón, J., Nakamura, M. 2009. Niches and distributional areas: concepts, methods, and assumptions. Proceedings of the National Academy of Sciences 106, 19644-19650. https://doi. org/10.1073/pnas.0901637106. 
Thuiller, W. 2003. Biomod - optimizing predictions of species distributions and projecting potential future shifts under global change. Global Change Biology 9, 1353-1362. https://doi. org/10.1046/j.1365-2486.2003.00666.x.

Thuiller, W., Georges, D., Engler, R. 2014. Biomod2: Ensemble platform for species distribution modeling.

Tylianakis, J.M., Didham, R.K., Bascompte, J., Wardle, D.A. 2008. Global change and species interactions in terrestrial ecosystems. Ecology Letters 11, 1351-1363. https://doi.org/10.1111/ j.1461-0248.2008.01250.x.

Van Kleunen, M., Weber, E., Fischer, M. 2010. A meta-analysis of trait differences between invasive and non-invasive plant species. Ecology Letters 13, 235-245. https://doi.org/10.1111/ j.1461-0248.2009.01418.x.

Zhu, G., Li, H., Zhao, L. 2016. Incorporating anthropogenic variables into ecological niche modeling to predict areas of invasion of Popillia japonica. Journal of Pest Science, 1-10. https:// doi.org/10.1007/s10340-016-0780-5. 


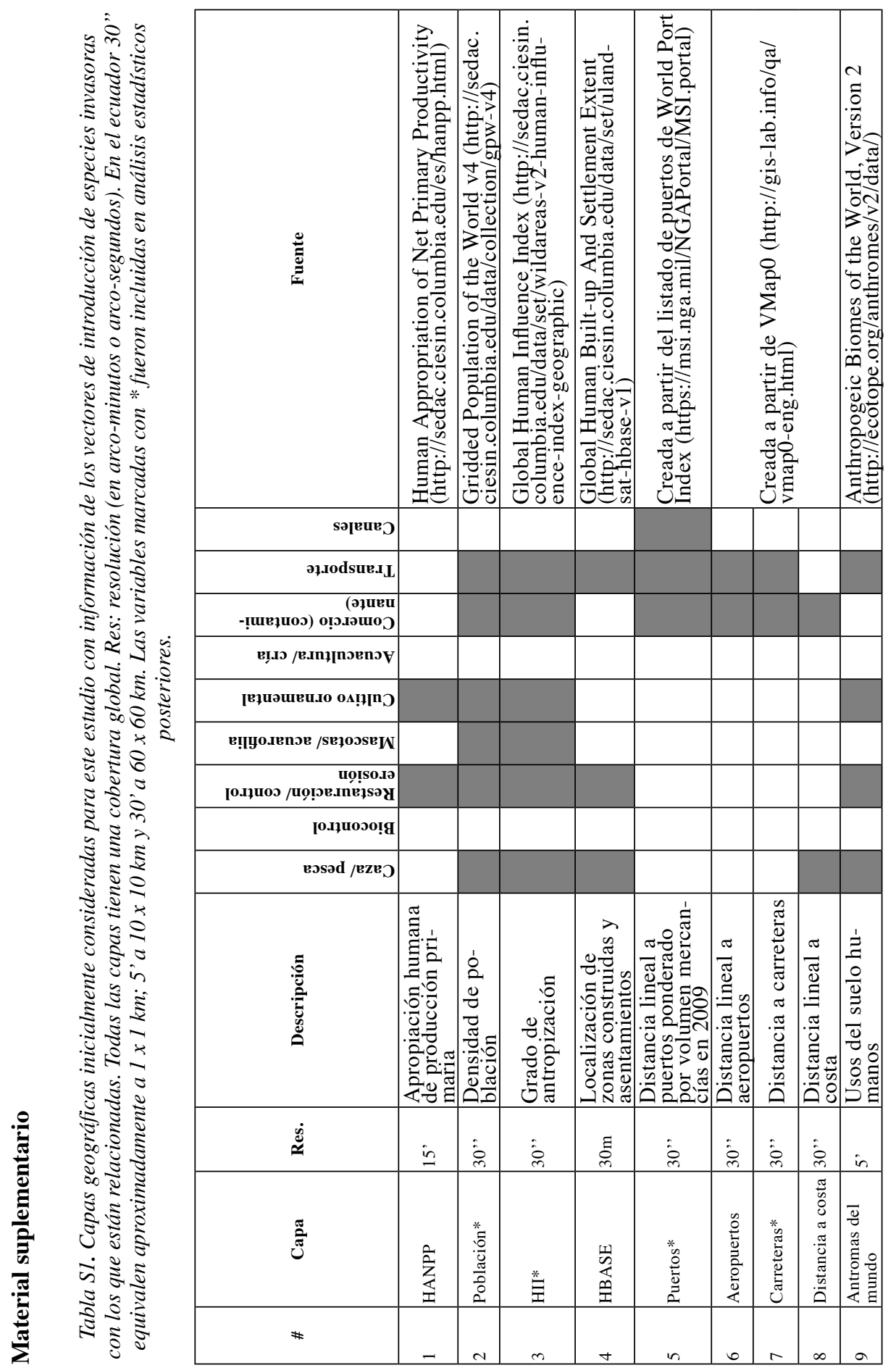




\begin{tabular}{|c|c|c|c|c|c|c|c|c|c|c|c|c|c|c|}
\hline 苞 & 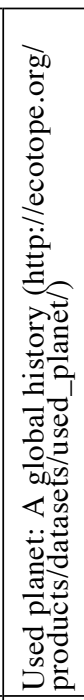 & 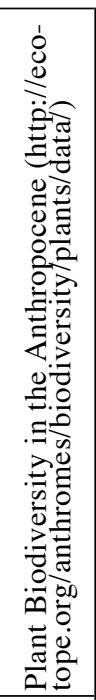 & \multicolumn{2}{|c|}{ 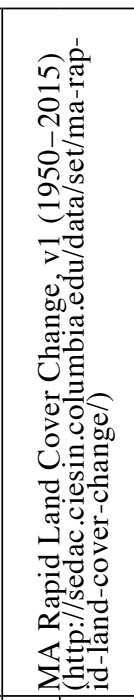 } & \multicolumn{2}{|c|}{  } & \multicolumn{3}{|c|}{ 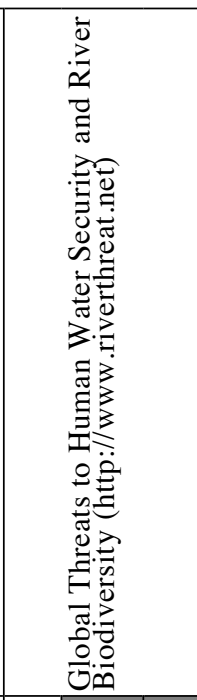 } & 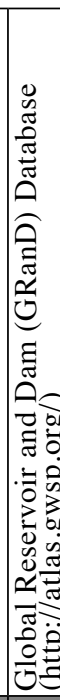 &  & 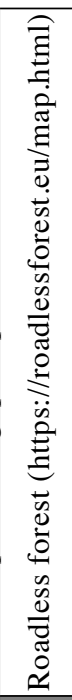 & 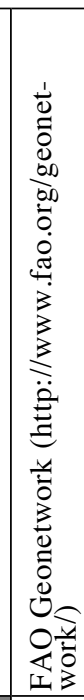 & 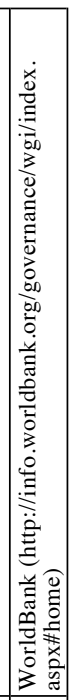 \\
\hline \multicolumn{15}{|c|}{ sә[вue } \\
\hline \multicolumn{15}{|c|}{ әр..10dsur.. $\mathbf{L}$} \\
\hline \multicolumn{15}{|c|}{  } \\
\hline \multicolumn{15}{|c|}{ 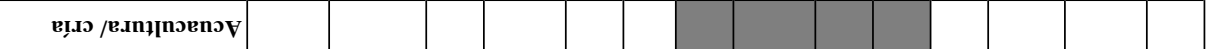 } \\
\hline \multicolumn{15}{|c|}{ 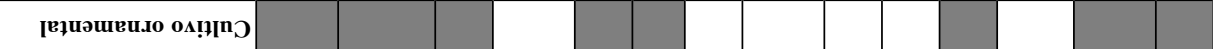 } \\
\hline \multicolumn{15}{|c|}{ в!!yo.denov /sełoose } \\
\hline \multicolumn{15}{|c|}{ 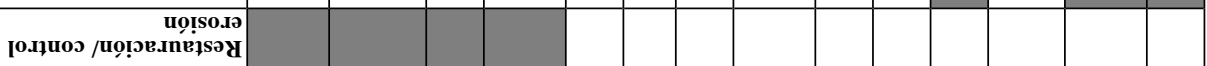 } \\
\hline \multicolumn{15}{|c|}{ [0.1૫u0so!g } \\
\hline \multicolumn{15}{|c|}{ eosəd / } \\
\hline 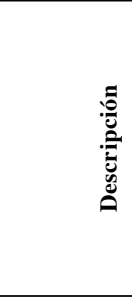 & 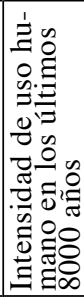 & 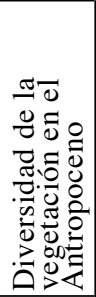 & 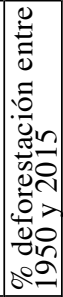 & 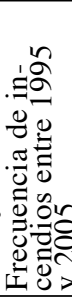 & 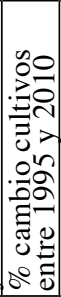 & 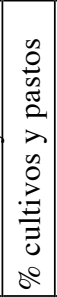 & 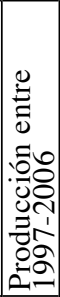 & 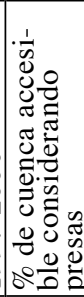 & 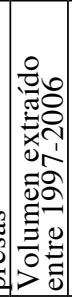 & 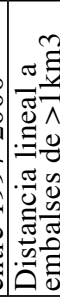 & 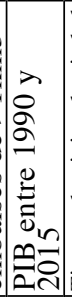 & 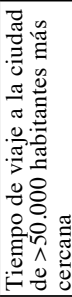 & 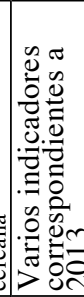 & 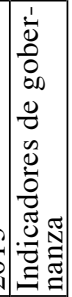 \\
\hline$\dot{\mathscr{n}}$ & i & in & $\dot{e}$ & $\dot{m}$ & in & in & $\dot{m}$ & ì & $\dot{m}$ & iे & $\dot{m}$ & iे & & \\
\hline שึّ & 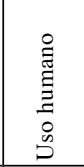 & 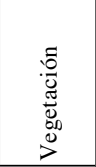 & 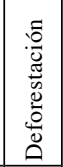 & 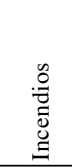 & $\begin{array}{l}\text { * } \\
0 \\
0 \\
\stackrel{\Xi}{\Xi} \\
\tilde{J}\end{array}$ & 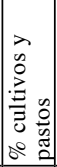 & 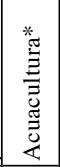 & 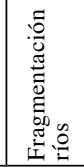 & $\begin{array}{c}\tilde{U} \\
0 \\
0 \\
0\end{array}$ & 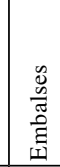 & $\cong$ & 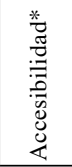 & $\frac{0}{4}$ & $\begin{array}{l}5 \\
3\end{array}$ \\
\hline$\$$ & 으 & $=$ & $\simeq$ & - & \pm & 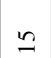 & $\underline{-1}$ & $\Xi$ & $\stackrel{\infty}{\simeq}$ & $\stackrel{2}{2}$ & సิ & $\vec{\lambda}$ & तี & $\pi$ \\
\hline
\end{tabular}


Tabla S2. Resultados de los Modelos de Nicho Ecológico calibrados con variables climáticas, integrando variables humanas relacionadas con la presión de propágulos, y cambio en los indicadores (modelo integrado-clima).

\begin{tabular}{|c|c|c|c|c|c|c|c|}
\hline \multirow{2}{*}{ Especies } & \multirow[b]{2}{*}{$\mathbf{N}$} & \multicolumn{2}{|c|}{ Clima } & \multicolumn{2}{|c|}{ Integrado } & \multicolumn{2}{|c|}{ Cambio } \\
\hline & & TSS & AUC & TSS & AUC & TSS & AUC \\
\hline Anguillicola crassus & 116 & 0,88 & 0,97 & 0,81 & 0,93 & $-0,06$ & $-0,04$ \\
\hline Cercopagis pengoi & 146 & 0,96 & 0,99 & 0,97 & 0,99 & 0,01 & 0,00 \\
\hline Corbicula fluminea & 2333 & 0,75 & 0,94 & 0,79 & 0,95 & 0,04 & 0,01 \\
\hline Cordylophora caspia & 143 & 0,87 & 0,97 & 0,86 & 0,97 & $-0,01$ & 0,00 \\
\hline Crassula helmsii & 1483 & 0,96 & 1,00 & 0,97 & 1,00 & 0,01 & 0,00 \\
\hline Dikerogammarus villosus & 498 & 0,97 & 0,99 & 0,95 & 0,99 & $-0,01$ & 0,00 \\
\hline Dreissena polymorpha & 3029 & 0,87 & 0,97 & 0,88 & 0,98 & 0,01 & 0,01 \\
\hline Elodea canadensis & 10463 & 0,89 & 0,98 & 0,88 & 0,98 & $-0,01$ & 0,00 \\
\hline Eriocheir sinensis & 443 & 0,87 & 0,98 & 0,92 & 0,98 & 0,05 & 0,01 \\
\hline Neogobius melanostomus & 482 & 0,91 & 0,99 & 0,91 & 0,99 & 0,00 & 0,00 \\
\hline Procambarus clarkii & 886 & 0,84 & 0,97 & 0,88 & 0,98 & 0,04 & 0,01 \\
\hline Pseudorasbora parva & 878 & 0,86 & 0,97 & 0,86 & 0,97 & 0,00 & 0,00 \\
\hline Salvelinus fontinalis & 3068 & 0,76 & 0,94 & 0,78 & 0,95 & 0,02 & 0,01 \\
\hline Aedes albopictus & 1410 & 0,74 & 0,93 & 0,80 & 0,96 & 0,06 & 0,02 \\
\hline Aphis gossypii & 559 & 0,60 & 0,87 & 0,65 & 0,90 & 0,06 & 0,03 \\
\hline Arion vulgaris & 835 & 0,94 & 0,99 & 0,96 & 0,99 & 0,02 & 0,00 \\
\hline Bemisia tabaci & 425 & 0,68 & 0,90 & 0,78 & 0,94 & 0,10 & 0,04 \\
\hline Cameraria ohridella & 1245 & 0,95 & 0,99 & 0,95 & 0,99 & 0,00 & 0,00 \\
\hline Ceratitis capitata & 705 & 0,70 & 0,91 & 0,71 & 0,92 & 0,01 & 0,01 \\
\hline Diabrotica virgifera & 583 & 0,88 & 0,98 & 0,89 & 0,98 & 0,01 & 0,00 \\
\hline Harmonia axyridis & 2285 & 0,84 & 0,97 & 0,86 & 0,98 & 0,02 & 0,01 \\
\hline Leptinotarsa decemlineata & 508 & 0,74 & 0,94 & 0,78 & 0,95 & 0,04 & 0,02 \\
\hline Linepithema humile & 1215 & 0,77 & 0,94 & 0,80 & 0,95 & 0,03 & 0,01 \\
\hline Liriomyza huidobrensis & 403 & 0,82 & 0,95 & 0,85 & 0,96 & 0,03 & 0,01 \\
\hline Spodoptera littoralis & 113 & 0,87 & 0,96 & 0,88 & 0,95 & 0,02 & 0,00 \\
\hline Acacia dealbata & 1277 & 0,74 & 0,93 & 0,79 & 0,95 & 0,05 & 0,02 \\
\hline Ailanthus altissima & 2566 & 0,89 & 0,98 & 0,89 & 0,99 & 0,00 & 0,00 \\
\hline Ambrosia artemisiifolia & 5212 & 0,77 & 0,94 & 0,81 & 0,96 & 0,04 & 0,01 \\
\hline Campylopus introflexus & 6143 & 0,77 & 0,95 & 0,80 & 0,96 & 0,03 & 0,01 \\
\hline Carpobrotus edulis & 773 & 0,87 & 0,97 & 0,87 & 0,98 & 0,00 & 0,00 \\
\hline Cortaderia selloana & 999 & 0,92 & 0,98 & 0,92 & 0,99 & 0,01 & 0,00 \\
\hline Echinocystis lobata & 1385 & 0,78 & 0,96 & 0,80 & 0,96 & 0,02 & 0,00 \\
\hline
\end{tabular}




\begin{tabular}{|l|l|l|l|l|l|l|l|}
\hline \multirow{2}{*}{ Especies } & \multicolumn{2}{|c|}{ Clima } & \multicolumn{2}{c|}{ Integrado } & \multicolumn{2}{c|}{ Cambio } \\
\cline { 2 - 8 } & \multicolumn{1}{|c|}{ N } & \multicolumn{1}{|c|}{ TSS } & AUC & TSS & AUC & TSS & AUC \\
\hline Fallopia japonica & 10723 & 0,70 & 0,91 & 0,71 & 0,90 & 0,01 & $-0,01$ \\
\hline Hedychium gardnerianum & 121 & 0,70 & 0,91 & 0,75 & 0,94 & 0,05 & 0,02 \\
\hline Heracleum mantegazzianum & 5633 & 0,63 & 0,89 & 0,82 & 0,96 & 0,19 & 0,07 \\
\hline Impatiens glandulifera & 8059 & 0,86 & 0,97 & 0,89 & 0,98 & 0,03 & 0,01 \\
\hline Opuntia ficus-indica & 369 & 0,90 & 0,99 & 0,92 & 0,99 & 0,02 & 0,01 \\
\hline Oxalis pes-caprae & 1397 & 0,52 & 0,81 & 0,77 & 0,94 & 0,24 & 0,13 \\
\hline Paspalum paspalodes & 2150 & 0,80 & 0,95 & 0,80 & 0,96 & 0,01 & 0,01 \\
\hline Prunus serotina & 6946 & 0,74 & 0,93 & 0,76 & 0,94 & 0,02 & 0,02 \\
\hline Rhododendron ponticum & 3182 & 0,91 & 0,99 & 0,91 & 0,99 & 0,00 & 0,00 \\
\hline Robinia pseudoacacia & 9365 & 0,83 & 0,97 & 0,83 & 0,97 & 0,01 & 0,00 \\
\hline Rosa rugosa & 5261 & 0,79 & 0,95 & 0,78 & 0,95 & 0,00 & 0,00 \\
\hline Branta canadensis & 40320 & 0,91 & 0,99 & 0,91 & 0,99 & 0,00 & 0,00 \\
\hline Cervus nippon & 1214 & 0,89 & 0,98 & 0,91 & 0,99 & 0,02 & 0,01 \\
\hline Lithobates catesbeianus & 4510 & 0,88 & 0,98 & 0,84 & 0,95 & $-0,03$ & $-0,03$ \\
\hline Mustela vison & 5301 & 0,88 & 0,97 & 0,89 & 0,98 & 0,01 & 0,00 \\
\hline Myocastor coypus & 2745 & 0,90 & 0,99 & 0,92 & 0,99 & 0,01 & 0,00 \\
\hline Nyctereutes procyonoides & 1772 & 0,93 & 0,98 & 0,89 & 0,96 & $-0,04$ & $-0,03$ \\
\hline Ondatra zibethicus & 3152 & 0,93 & 0,99 & 0,95 & 1,00 & 0,02 & 0,00 \\
\hline Oxyura jamaicensis & 17144 & 0,93 & 0,99 & 0,95 & 0,99 & 0,01 & 0,00 \\
\hline Procyon lotor & 15468 & 0,79 & 0,96 & 0,80 & 0,96 & 0,01 & 0,00 \\
\hline Psittacula krameri & 1279 & 0,88 & 0,98 & 0,88 & 0,98 & 0,00 & 0,00 \\
\hline Rattus norvegicus & 9825 & 0,77 & 0,94 & 0,80 & 0,95 & 0,03 & 0,01 \\
\hline Sciurus carolinensis & 5166 & 0,80 & 0,96 & 0,78 & 0,95 & $-0,02$ & 0,00 \\
\hline Tamias sibiricus & 131 & 0,97 & 1,00 & 0,98 & 1,00 & 0,01 & 0,00 \\
\hline Threskiornis aethiopicus & 2347 & 0,86 & 0,98 & 0,87 & 0,98 & 0,01 & 0,00 \\
\hline Trachemys scripta & 1503 & 0,93 & 0,99 & 0,93 & 0,99 & 0,01 & 0,00 \\
\hline
\end{tabular}






Travel time to major cities

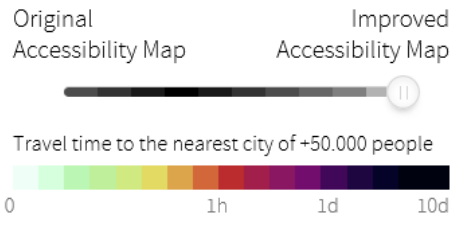

Figura S1. Mapa de accesibilidad en Europa. La accesibilidad se mide como "tiempo de viaje a la ciudad de $>50.000$ habitantes más cercana" y por tanto a menor valor (colores brillantes), mayor accesibilidad. Las zonas de mayor influencia humana incluyen zonas costeras, grandes ciudades y alrededores del canal de la Mancha (Inglaterra, norte de Francia, Bélgica y Países

Bajos). Por su parte, las zonas más prístinas corresponden a Península noruega, norte de Escocia, países del Este y zonas de alta montaña (Pirineos, Alpes). Mapa extraído de https:// roadlessforest.eu/map.html 